\title{
THREE TYPES OF SUPERPOTENTIALS FOR PERTURBATIONS IN THE EINSTEIN-GAUSS-BONNET GRAVITY
}

\author{
A.N.Petrov \\ Relativistic Astrophysics group, Sternberg Astronomical institute, \\ Universitetskii pr., 13, Moscow, 119992, RUSSIA \\ e-mail: anpetrov@rol.ru
}

telephone number: $+7(495) 7315222$

fax: $+7(495) 9328841$

PACS numbers: 04.50.+h, 04.20.Cv, 04.20.Fy

\begin{abstract}
Superpotentials (antisymmetric tensor densities) in Einstein-Gauss-Bonnet (EGB) gravity for arbitrary types of perturbations on arbitrary curved backgrounds are constructed. As a basis, the generalized conservation laws in the framework of an arbitrary D-dimensional metric theory, where conserved currents are expressed through divergences of superpotentials, are used. Such a derivation is exact (perturbations are not infinitesimal) and is approached, when a one solution (dynamical) is considered as a perturbed system with respect to another solution (background). Three known prescriptions are elaborated: these are the canonical Nœther theorem, the Belinfante symmetrization rule and the field-theoretical derivation. All the three approaches are presented in an unique way convenient for comparisons and a development. Exact expressions for the 01-component of the three types of the superpotentials are derived in the case, when an arbitrary static Schwarzschild-like solution in the EGB gravity is considered as a perturbed system with respect to a background of the same type. These formulae are used for calculating the mass of the Schwarzschild-anti-de Sitter black hole in the EGB gravity. As a background both the anti-de Sitter spacetime in arbitrary dimensions and a not maximally symmetric "mass gap" vacuum in 5 dimensions are considered. Problems and perspectives for a future development, including the Lovelock gravity, are discussed.
\end{abstract}




\section{Introduction}

Multidimensional (multi-D) metric theories of gravity become very popular (see, for example, reviews [1] - [6] and references there in). It is important to construct conserved quantities for perturbations in such theories. Several useful and interesting approaches were presented (see, for example, [7] - [10]). However, because these theories and their solutions are very complicated and have nontrivial properties, one needs to expand a research of conservation laws. In particular, curved backgrounds (including not symmetrical, etc.) are usual, therefore a construction of covariant conserved quantities on such backgrounds is necessary. Already, in 4-dimensional general relativity (4D GR), there exist approaches satisfying this requirement. From them three ones are more universal and satisfy necessary tests in 4D GR, when one calculates masses of black holes, energy and momentum fluxes in the Bondi-Sachs solution, etc.. It is natural to develop these three approaches in multi-D theories.

One of the approaches, canonical, begins from the Einstein pseudotensor [11] and the Freud superpotential [12]. Its generalized form in 4D GR is presented by Katz, Bičák and Lynden-Bell (KBL) [13]. The second approach is based on the Belinfante symmetrization method [14], which firstly in 4D GR has been applied by Papapetrou [15] for symmetrization of the Einstein pseudotensor. A generalized application of the Belinfante method in 4D GR is presented in [16]. At last, the third approach starts from the linearization of the Einstein equations and treating all the other terms as an effective energy-momentum [17]. This picture has been recreated in the form of the Lagrangian based field theory for perturbations on a flat background by Deser [18], so-called field-theoretical derivation, which later has been developed for arbitrary curved backgrounds [19] - [22]. All the three methods derive the exact (not infinitesimal or approximate) perturbations. Such a derivation is approached when a one solution (dynamical) of the theory is considered as a perturbed system with respect to another solution (background) of the same theory. In the present paper we follow just this scheme and present exact formulae. Linear or of higher order approximations easily follow if the exact form is presented.

In multi-D theories, the above mentioned approaches already have received a development as follows. In the works [23] - [25] Deruelle and Katz with coauthors successfully develop the canonical KBL approach [13]. The method based on the Belinfante procedure is continued in our works [26, 22]. The field-theoretical derivation in multi-D arbitrary gravities starts in [27]. In the recent works [28] - [30] Deser and Tekin, applying the Abbott-Deser procedure [19], suggest a construction of conserved charges for perturbations about vacua in quadratic (in curvature) theories. A development of our [27] and the Deser-Tekin [28] methods is continued in [31]. The results in [23] - [31] show that all the three approaches are perspective in constructing conserved charges for numerous known and new solutions in multi-D theories.

Among multi-D theories, the Gauss-Bonnet modification (quadratic order of the Lovelock type [32]) of the Einstein theory takes a very important place. The main goal of the present paper is to construct superpotentials of the most general form in the framework of this theory with using the aforementioned approaches. As a basis, we use the results obtained in [22] in the framework of an arbitrary multi-D metric theory. Following them, in the next section 2, we derive out the explicit generalized expressions for the superpotentials. In section 3, we construct superpotentials in the Einstein-Gauss-Bonnet (EGB) gravity by

all the three methods for arbitrary types of perturbations on arbitrary curved backgrounds. Thus many new solutions (see, e.g., [33] - [43]) can be examined. Concerning the canonical method, we in many lines repeat the work [23], however, we present it in the unique style of the present paper. In section 4, the developed formalism is used for an examination of static spherically symmetric solutions of the Schwarzschild-like type 
in EGB gravity. In spite of a visible simplicity, such solutions are very rich in interesting properties. For calculating a mass we derive out exact expressions for the 01-component of the superpotentials of all the three types, considering background solutions of the same Schwarzschild-like form. In section 5, for each of the approaches the mass of the Schwarzschild-anti-de Sitter (S-AdS) black hole (BH) [44] is calculated. In the first case, as a usual background we consider the AdS spacetime in arbitrary D dimensions. The second case is not trivial, in 5 dimensions as a not maximally symmetric background we consider the vacuum of a "mass gap" (see, e.g., [4]). All the results are acceptable. In section 6, problems of the methods and perspectives for a future development are discussed.

\section{Preliminaries}

In this section, we shortly represent the results of the section in [22] related to constructing superpotentials in an arbitrary multi-D metric theory. Consider the Lagrangian:

$$
\hat{\mathcal{L}}_{D}=-\frac{1}{2 \kappa} \hat{\mathcal{L}}_{g}\left(g^{a}\right)+\hat{\mathcal{L}}_{m}\left(g^{a}, \Phi\right)
$$

including derivatives up to the second order of $g^{a}$ and $\Phi$. An independent metric variable $g^{a}$ is thought as a one defined in the set

$$
g^{a}=\left\{g^{\mu \nu}, g_{\mu \nu}, \sqrt{-g} g^{\mu \nu}, \sqrt{-g} g_{\mu \nu},(-g) g^{\mu \nu}, \ldots\right\}
$$

where the Greek indexes numerate $D$-dimensional spacetime coordinates. Variation of (2.1) with respect to each $g^{a}$ leads to the gravitational equations, which are equivalent between themselves. Below we will explain why it is important to examine the different definitions (2.2). Matter sources without concretization are denoted as $\Phi$; here and below "hat" means densities of the wight $+1 ;(, \alpha) \equiv \partial_{\alpha}$ means ordinary derivatives.

Our goal is to describe perturbations on a background of a fixed system. For the last we assume the background $D$-dimensional spacetime with the metric $\bar{g}_{\mu \nu}$, on the basis of which the background Christoffel symbols $\bar{\Gamma}_{\tau \rho}^{\sigma}$, covariant derivatives $\bar{D}_{\alpha}$ and the background Riemannian tensor $\bar{R}_{\tau \rho \pi}^{\sigma}$ are constructed; here and below "bar" means that a quantity is a background one. We use also the background Lagrangian defined as $\overline{\hat{\mathcal{L}}}_{D}=\hat{\mathcal{L}}_{D}\left(\bar{g}^{a}, \bar{\Phi}\right)$ and corresponding background gravitational equations. We set that the background fields $\bar{g}^{a}$ and $\bar{\Phi}$ satisfy the background equations, and, thus are known (fixed).

Using different combinations of the pure gravitational Lagrangians $-\hat{\mathcal{L}}_{g} / 2 \kappa$ and $-\overline{\hat{\mathcal{L}}}_{g} / 2 \kappa$ and applying the Nœther procedure directly or in a combination with other methods one obtains the correspondent identities of the unique form:

$$
\hat{\mathcal{I}}^{\alpha}(\xi) \equiv \partial_{\beta} \hat{\mathcal{I}}^{\alpha \beta}(\xi)
$$

where $\xi^{\alpha}$ is a displacement vector, $\hat{\mathcal{I}}^{\alpha \beta}$ is an antisymmetric tensor density, thus $\bar{D}_{\alpha \beta} \hat{\mathcal{I}}^{\alpha \beta}(\xi) \equiv \partial_{\alpha \beta} \hat{\mathcal{I}}^{\alpha \beta}(\xi) \equiv 0$; and $\hat{\mathcal{I}}^{\alpha}$ is a vector density. After substitution of the dynamical and background equations into $\hat{\mathcal{I}}^{\alpha}$ it acquires the sense of the conserved current $\bar{D}_{\alpha} \hat{\mathcal{I}}^{\alpha}(\xi)=\partial_{\alpha} \hat{\mathcal{I}}^{\alpha}(\xi)=0$, and the identity (2.3) acquires the sense of the conservation law (already not identity) with the superpotential $\hat{\mathcal{I}}^{\alpha \beta}$. Integrating the aforementioned differential conservation laws and using the D-dimensional Gauss theorem, one can obtain the integral charges in a generalized form:

$$
\mathcal{P}(\xi)=\int_{\Sigma} d^{D-1} x \hat{\mathcal{I}}^{0}(\xi)=\oint_{\partial \Sigma} d S_{i} \hat{\mathcal{I}}^{0 i}(\xi)
$$

where $\Sigma$ is a spatial $(D-1)$-dimensional hypersurface $x^{0}=$ const, $\partial \Sigma$ is its $(D-2)$-dimensional boundary, zero's and small Latin indexes numerate time and space coordinates, respectively. In depending on boundary 
conditions at $\partial \Sigma$ (or on a falloff of potentials, if $\partial \Sigma$ presents infinity), the integral (2.4) defines the conserved on $\Sigma$ quantity $\partial_{0} \mathcal{P}(\xi)=0$ or expresses its flux through $\partial \Sigma$ : $\partial_{0} \mathcal{P}(\xi)=-\oint_{\partial \Sigma} d S_{i} \hat{\mathcal{I}}^{i}(\xi)$.

As a result of an application of the Nœther theorem the quantities in (2.3) and (2.4) in general contain arbitrary displacement vectors $\xi^{\alpha}$. However, an interpretation such quantities, as a rule, is impossible or not understandable $^{1}$, whereas using the Killing vectors lead to the clear treating. Here, to define the energy/mass of the system in our applications we use the timelike Killing vector of the background.

\subsection{The canonical superpotentials}

To construct the canonical expressions we follow the KBL ideology [13] and consider the Lagrangian:

$$
\hat{\mathcal{L}}_{G}=-\frac{1}{2 \kappa}\left(\hat{\mathcal{L}}_{g}-\overline{\hat{\mathcal{L}}}_{g}+\partial_{\alpha} \hat{d}^{\alpha}\right),
$$

which, of course, leads to the usual gravitational equations. Then the background metric $\bar{g}_{\mu \nu}$ is incorporated into $\hat{\mathcal{L}}_{g}$ by the usual way as follows. The ordinary derivatives $\partial_{\alpha}$ are rewritten over the covariant $\bar{D}_{\alpha}$ ones by changing $\partial_{\tau} g^{a} \equiv \bar{D}_{\tau} g^{a}-\left.\bar{\Gamma}_{\tau \rho}^{\sigma} g^{a}\right|_{\sigma} ^{\rho}$ where $\left.g^{a}\right|_{\beta} ^{\alpha}$ is defined by the transformation properties of $g^{a}$. Then, with using

$$
\begin{aligned}
\Delta_{\mu \nu}^{\alpha} & =\Gamma_{\mu \nu}^{\alpha}-\bar{\Gamma}_{\mu \nu}^{\alpha}=\frac{1}{2} g^{\alpha \rho}\left(\bar{D}_{\mu} g_{\rho \nu}+\bar{D}_{\nu} g_{\rho \mu}-\bar{D}_{\rho} g_{\mu \nu}\right), \\
R_{\tau \rho \sigma}^{\lambda} & =\bar{D}_{\rho} \Delta_{\tau \sigma}^{\lambda}-\bar{D}_{\sigma} \Delta_{\tau \rho}^{\lambda}+\Delta_{\rho \eta}^{\lambda} \Delta_{\tau \sigma}^{\eta}-\Delta_{\eta \sigma}^{\lambda} \Delta_{\tau \rho}^{\eta}+\bar{R}_{\tau \rho \sigma}^{\lambda}
\end{aligned}
$$

we transform the pure metric Lagrangian $\hat{\mathcal{L}}_{g}$ into an explicitly covariant form: $\hat{\mathcal{L}}_{g}=\hat{\mathcal{L}}_{c}=\hat{\mathcal{L}}_{c}\left(g^{a} ; \bar{D}_{\alpha} g^{a} ; \bar{D}_{\beta} \bar{D}_{\alpha} g^{a}\right)$. Then, a direct application of the canonical Nœether procedure to $-\hat{\mathcal{L}}_{c} / 2 \kappa$ gives the identity of the type (2.3) with the superpotential:

$$
\hat{\imath}_{C}^{\alpha \beta}(\xi)=\left(\frac{2}{3} \bar{D}_{\lambda} \hat{n}_{\sigma}{ }^{[\alpha \beta] \lambda}-\hat{m}_{\sigma}^{[\alpha \beta]}\right) \xi^{\sigma}-\frac{4}{3} \hat{n}_{\sigma}^{[\alpha \beta] \lambda} \bar{D}_{\lambda} \xi^{\sigma}
$$

where the coefficients are calculated with the use of the general formulae:

$$
\begin{aligned}
\hat{m}_{\sigma}{ }^{\alpha \tau} & \equiv-\frac{1}{2 \kappa}\left\{\left.\left[\frac{\partial \hat{\mathcal{L}}_{c}}{\partial\left(\bar{D}_{\alpha} g^{a}\right)}-\bar{D}_{\beta}\left(\frac{\partial \hat{\mathcal{L}}_{c}}{\partial\left(\bar{D}_{\beta} \bar{D}_{\alpha} g^{a}\right)}\right)\right] g^{a}\right|_{\sigma} ^{\tau}\right. \\
& \left.-\frac{\partial \hat{\mathcal{L}}_{c}}{\partial\left(\bar{D}_{\tau} \bar{D}_{\alpha} g^{a}\right)} \bar{D}_{\sigma} g^{a}+\frac{\partial \hat{\mathcal{L}}_{c}}{\partial\left(\bar{D}_{\beta} \bar{D}_{\alpha} g^{a}\right)} \bar{D}_{\beta}\left(\left.g^{a}\right|_{\sigma} ^{\tau}\right)\right\}, \\
\hat{n}_{\sigma}{ }^{\alpha \tau \beta} & \equiv-\frac{1}{4 \kappa}\left[\left.\frac{\partial \hat{\mathcal{L}}_{c}}{\partial\left(\bar{D}_{\beta} \bar{D}_{\alpha} g^{a}\right)} g^{a}\right|_{\sigma} ^{\tau}+\left.\frac{\partial \hat{\mathcal{L}}_{c}}{\partial\left(\bar{D}_{\tau} \bar{D}_{\alpha} g^{a}\right)} g^{a}\right|_{\sigma} ^{\beta}\right] .
\end{aligned}
$$

To calculate the full superpotential related to the Lagrangian (2.5) one has to apply the barred procedure to $(2.8)$ and the Nœther procedure to the divergence in (2.5):

$$
\hat{\mathcal{I}}_{C}^{\alpha \beta}(\xi)=\hat{\imath}_{C}^{\alpha \beta}(\xi)-\overline{\hat{\imath}_{C}^{\alpha \beta}(\xi)}+\kappa^{-1} \xi^{[\alpha} \hat{d}^{\beta]} .
$$

As is seen, if one adds different divergences to the Lagrangian (2.5), different canonical superpotentials (2.11) appear. Such a situation is natural and could be interesting in gravitational theory, when analogies, for example, with thermodynamics are carried out [45]. There is no a crucial principle for a definition of divergences, however the Silva method [46] looks as a perspective criterium. The boundary conditions

\footnotetext{
${ }^{1}$ Nevetherless, sometimes one can find out a reasonable using even non-Killing vectors, like in [16], where conformal Killing vectors were used for derivation of perturbations on the Friedmann-Robertson-Walker background.
} 
for deriving superpotentials in [46] could be imposed by including a correspondent divergence into the Lagrangian. This divergence is defined by an unique way. Thus, supposing the boundary conditions for an isolated system in 4D GR the Silva method shows that the KBL divergence [13] is the unique answer for the Dirichlet boundary conditions.

The superpotential (2.11) with the KBL divergence in the Lagrangian derived for the 4D Einstein theory, simplified to the Minkowski background in the Cartesian coordinates and with the translation Killing vectors $\xi^{\alpha} \rightarrow \lambda_{(\beta)}^{\alpha}=\delta_{\beta}^{\alpha}$ is just the very known Freud superpotential [12]. The current corresponding to (2.11) under these simplifications transforms into the famous Einstein pseudotensor [11].

In a recent work [47], Katz and Livshits develop Silva's method applying it in the Lovelock theory [32] in the first order formalism (Palatini presentation). Using boundary conditions correspondent to an isolated system, they derive out equations for superpotentials, which are easily integrated giving the general expressions for all the superpotentials associated with the Lovelock Lagrangians. In particular, in multi-D

GR, the Katz-Livshits superpotential turns out uniquely the KBL superpotential; in EGB gravity, they present explicitly a new superpotential. The last naturally transfers into the KBL superpotential for $D=4$. Below we are interested in a divergence induced by the Katz-Livshits procedure in the EGB Lagrangian. Together with this we consider the divergence presented by Deruelle, Katz and Ogushi [23], which has been derived "in such a way that the boundary term [after variation] does not contain terms proportional to normal derivatives of the metric variations".

\subsection{The Belinfante symmetrization procedure}

The application of the generalized Belinfante procedure $[16,22]$ to the canonical conserved laws with the superpotential (2.8) leads to the superpotential:

$$
\hat{\imath}_{B}^{\alpha \beta}(\xi)=2\left(\frac{1}{3} \bar{D}_{\rho} \hat{n}_{\sigma}^{[\alpha \beta] \rho}+\bar{D}_{\tau} \hat{n}_{\lambda}{ }^{\tau \rho[\alpha} \bar{g}^{\beta] \lambda} \bar{g}_{\rho \sigma}\right) \xi^{\sigma}-\frac{4}{3} \hat{n}_{\sigma}^{[\alpha \beta] \lambda} \bar{D}_{\lambda} \xi^{\sigma} .
$$

Fully the superpotential correspondent to the Lagrangian (2.5) has the form:

$$
\hat{\mathcal{I}}_{B}^{\alpha \beta}(\xi)=\hat{\imath}_{B}^{\alpha \beta}(\xi)-\overline{\hat{\imath}_{B}^{\alpha \beta}(\xi)} .
$$

Because this superpotential depends on the $n$-coefficients only it vanishes for Lagrangians with only the first order derivatives (see (2.10)) and is well adapted to theories with second derivatives in Lagrangians, like quadratic [28] or of the Lovelock type [32] theories.

The superpotential (2.13) and the correspondent current derived for the $4 \mathrm{D}$ GR, simplified to the Minkowski background in the Cartesian coordinates and with the translation Killing vectors are the well known famous Papapetrou superpotential and its current [15].

\subsection{The field-theoretical prescription}

Turning to the field-theoretical approach one has to use the decomposition of the metric and matter variables

$$
g^{a}=\bar{g}^{a}+h^{a}, \quad \Phi=\bar{\Phi}+\phi .
$$

Following $[27]$ we describe the perturbed system by the Lagrangian:

$$
\hat{\mathcal{L}}_{D}^{d y n}=\hat{\mathcal{L}}_{D}(\bar{g}+h, \bar{\Phi}+\phi)-h^{a} \frac{\delta \overline{\hat{\mathcal{L}}}_{D}}{\delta \bar{g}^{a}}-\phi \frac{\delta \overline{\hat{\mathcal{L}}}_{D}}{\delta \bar{\Phi}}-\overline{\hat{\mathcal{L}}}_{D}+\text { div } .
$$


Subscript "dyn" is used because the perturbations $h^{a}$ and $\phi$ play now the role of the dynamical fields. The background equations should not be taken into account before variation of $\hat{\mathcal{L}}_{D}^{d y n}$ with respect to $\bar{g}^{a}$ and $\bar{\Phi}$. Here, we need in the gravitational equations related to the Lagrangian (2.15) only on a vacuum background $\left(\overline{\hat{\mathcal{L}}}_{m}=0\right)$ :

$$
\hat{G}_{\mu \nu}^{L}=\kappa \hat{t}_{\mu \nu} .
$$

They are equivalent to the equations in the usual form related to the Lagrangian (2.1). The linear in $h^{a}$ expression and the generalized symmetric energy-momentum of the perturbations $h^{a}$ and $\phi$ are defined as

$$
\hat{G}_{\mu \nu}^{L} \equiv \frac{\delta}{\delta \bar{g}^{\mu \nu}} h^{a} \frac{\delta \overline{\hat{\mathcal{L}}}_{g}}{\delta \bar{g}^{a}}, \quad \hat{t}_{\mu \nu} \equiv 2 \frac{\delta \hat{\mathcal{L}}_{D}^{d y n}}{\delta \bar{g}^{\mu \nu}} .
$$

With taking into account the gravitational background equations one can rewrite the left hand side of (2.16) with another independent gravitational variables, instead of $h^{a}$ :

$$
\hat{l}_{(a)}^{\mu \nu} \equiv h^{a} \partial \overline{\hat{g}}^{\mu \nu} / \partial \bar{g}^{a} .
$$

Usually to construct superpotentials one contracts the left hand side of (2.16) with the background Killing vectors and provides direct algebraic transformations, like in [19, 28]. We present a more universal way $[22,31]$ as follows. Conserved quantities can be obtained and described analyzing only the scalar density

$$
\hat{\mathcal{L}}_{1} \equiv-\frac{1}{2 \kappa} h^{a} \frac{\delta \overline{\hat{\mathcal{L}}}_{g}}{\delta \bar{g}^{a}} \equiv-\frac{1}{2 \kappa} \hat{l}_{(a)}^{\alpha \beta} \frac{\delta \overline{\hat{\mathcal{L}}}_{g}}{\delta \overline{\hat{g}}^{\alpha \beta}},
$$

which is the linear pure metric term in the Lagrangian (2.15). After applying the Nother procedure directly and some algebraic transformations one obtains the superpotential:

$$
\hat{\mathcal{I}}_{S}^{\alpha \beta} \equiv \frac{4}{3}\left(2 \xi^{\sigma} \bar{D}_{\lambda} \hat{N}_{\sigma}^{[\alpha \mid \beta] \lambda}-\hat{N}_{\sigma}^{[\alpha \mid \beta] \lambda} \bar{D}_{\lambda} \xi^{\sigma}\right) \equiv \frac{8}{3} \bar{D}_{\lambda}\left(\hat{N}_{\sigma}^{[\alpha \mid \beta] \lambda} \xi^{\sigma}\right)-4 \hat{N}_{\sigma}^{[\alpha \mid \beta] \lambda} \bar{D}_{\lambda} \xi^{\sigma}
$$

where

$$
\hat{N}^{\rho \lambda \mid \mu \nu}=\partial \hat{\mathcal{L}}_{1} / \partial \bar{g}_{\rho \lambda, \mu \nu} .
$$

Conservation laws in the field-theoretical approach are based on the equations (2.16) with the symmetrical energy-momentum in (2.17), therefore we use the subscript " $S$ ".

To compare the expressions of this subsection with the known ones in 4D GR we again could do simplifications, like in previous subsections. Then, the equations (2.16) in varibles $\hat{l}^{\mu \nu}=\hat{g}^{\mu \nu}-\overline{\hat{g}^{\mu \nu}}$ repeat the equations presented by Weinberg [17], the superpotential (2.20) again transforms into the Papapetrou superpotential [15], note that in the 4D GR the Belinfante and the field-theoretical approaches give the same result [22]. Under more weak restrictions, say, to AdS/dS backgrounds in 4D GR the superpotential (2.20) goes to the Abbott-Deser expression [19]. At last, the expression (2.20) generalizes both our superpotential in 4D GR [16] and the Deser-Tekin expressions [28] in quadratic theories, if there one considers equations not more than of the second order.

\subsection{Instant remarks}

The first remark is of general type. Keeping in mind cosmological and astrophysical applications, conservation laws of the type (2.3) and (2.4) could play an important role to connect non-local conserved quantities (surface integrals) with local ones (currents for perturbations). 
The other remarks are as follow. 1) The canonical and Belinfante corrected approaches are bimetric. After substituting the decompositions (2.14) into the superpotentials (2.11) and (2.13) they are presented in the explicitly perturbed form, like (2.20). 2) Here, we consider theories, equations of which have derivatives not higher than of second order only, like the Lovelock theories [32]. However our results can be easily generalized for theories with derivatives of higher orders both in Lagrangians and in equations. Thus we agree with the discussion in the paper [48] related to our results [31]. 3) Viewing all the three types of the superpotentials (2.11), (2.13) and (2.20), it is important to note that their form is left the same both for vacuum and for nonvacuum backgrounds, and both for Killing and for arbitrary displacement vectors. 4) Unlike the canonical quantities, the Belinfante corrected and the field-theoretical superpotentials do not depend on divergences at all that could be necessary in various situations. 5) All the presented here procedures give well defined superpotentials in the sense that they are uniquely defined by the Lagrangian.

\section{Superpotentials in the EGB gravity}

Here, applying the formulae of the above section we derive out superpotentials in the EGB gravity presented by the action:

$$
S=-\frac{1}{2 \kappa} \int d^{D} x \hat{\mathcal{L}}_{E G B}=-\frac{1}{2 \kappa} \int d^{D} x \sqrt{-g}\left[R-2 \Lambda_{0}+\alpha\left(R_{\mu \nu \rho \sigma}^{2}-4 R_{\mu \nu}^{2}+R^{2}\right)\right],
$$

$\kappa=2 \Omega_{D-2} G_{D}>0$ and $\alpha>0 ; G_{D}$ is the $D$-dimensional Newton constant, and we restrict ourselves by $\Lambda_{0} \leq 0$. Below, the subscripts " $E$ " is related to the pure Einstein part in (3.1), and the subscript " $G B$ " is related to the Gauss-Bonnet part with the connection constant $\alpha$.

At first we calculate the canonical superpotential (2.11). With the use of (2.6) and (2.7) we represent $\hat{\mathcal{L}}_{E G B}$ into a covariantized form with the external background metric. After that we calculate the coefficients (7.4) and (2.10), substitute them into (2.8) and obtain

$$
\begin{aligned}
\hat{\imath}_{C}^{\alpha \beta} & =E^{\hat{\imath}_{C}^{\alpha \beta}}+{ }_{G B} \hat{\imath}_{C}^{\alpha \beta} \\
& =\frac{1}{\kappa}\left(\hat{g}^{\rho[\alpha} \bar{D}_{\rho} \xi^{\beta]}+\hat{g}^{\rho[\alpha} \Delta_{\rho \sigma}^{\beta]} \xi^{\sigma}\right) \\
& +\frac{\alpha \sqrt{-g}}{\kappa}\left\{6 R_{\tau}^{[\alpha \beta] \rho} \Delta_{\sigma \rho}^{\tau}-2 R_{\sigma}{ }^{\rho \tau[\alpha} \Delta_{\rho \tau}^{\beta]}-6 R^{\rho[\alpha \beta]}{ }_{\sigma} \Delta_{\tau \rho}^{\tau}+4 R^{\rho[\alpha} \Delta_{\sigma \rho}^{\beta]}-2 R_{\sigma}^{\rho} \Delta_{\rho \tau}^{[\alpha} g^{\beta] \tau}\right. \\
& -4 R^{\rho \tau} \delta_{\sigma}^{[\alpha} \Delta_{\rho \tau}^{\beta]}+2 R^{\rho[\alpha} g^{\beta] \tau} \Delta_{\rho \tau}^{\pi} g_{\sigma \pi}+8 R_{\rho}^{[\alpha} g^{\beta] \tau} \Delta_{\sigma \tau}^{\rho}+8 R_{\sigma}^{[\alpha} g^{\beta] \rho} \Delta_{\tau \rho}^{\tau}+4 R_{\sigma}^{[\alpha} \Delta_{\tau \rho}^{\beta]} g^{\tau \rho}+4 R_{\sigma}^{[\alpha} g^{\beta] \rho} \Delta_{\tau \rho}^{\tau} \\
& \left.-2 R \Delta_{\sigma \rho}^{[\alpha} g^{\beta] \rho}-4 R \Delta_{\tau \rho}^{\tau} \delta_{\sigma}^{[\alpha} g^{\beta] \rho}+\bar{D}_{\tau} R_{\sigma}{ }^{\tau \beta}+6 g_{\sigma \rho} g^{\tau[\alpha} \bar{D}_{\tau} R^{\beta] \rho}+2 \delta_{\sigma}^{[\alpha} g^{\beta] \rho} \bar{D}_{\rho} R\right\} \xi^{\sigma} \\
& -\frac{2 \alpha \sqrt{-g}}{\kappa}\left\{R_{\sigma}{ }^{\lambda \alpha \beta}+4 g^{\lambda[\alpha} R_{\sigma}^{\beta]}+\delta_{\sigma}^{[\alpha} g^{\beta] \lambda} R\right\} \bar{D}_{\lambda} \xi^{\sigma} .
\end{aligned}
$$

The barred expression is derived from the above and is simpler because $\bar{\Delta}_{\alpha \beta} \equiv 0$ :

$$
\begin{aligned}
\overline{\hat{\imath}_{C}^{\alpha \beta}} & =E^{\overline{\hat{\imath}^{\alpha \beta}}}+{ }_{G B} \overline{\hat{\imath}_{C}^{\alpha \beta}} \\
& =\frac{1}{\kappa} \overline{D^{[\alpha} \hat{\xi}^{\beta]}} \\
& +\frac{\alpha}{\kappa}\left\{\overline{D_{\tau} \hat{R}_{\sigma}}{ }^{\tau \beta}+6 \overline{D^{[\alpha} \hat{R}_{\sigma}^{\beta]}}+2 \delta_{\sigma}^{[\alpha} \overline{D^{\beta]} \hat{R}}\right\} \xi^{\sigma} \\
& -\frac{2 \alpha}{\kappa}\left\{\overline{\hat{R}}_{\sigma}^{\lambda \alpha \beta}+4 \overline{g^{\lambda[\alpha} \hat{R}_{\sigma}^{\beta]}}+\delta_{\sigma}^{[\alpha} \overline{g^{\beta] \lambda} \hat{R}}\right\} \bar{D}_{\lambda} \xi^{\sigma} .
\end{aligned}
$$

To finalize constructing the superpotential (2.11) one needs to fix a divergence in the Lagrangian (2.5). As we promised, we consider two possibilities. At first, we follow the recommendation in [23]: $\hat{d}_{D K O}^{\lambda}=$ 
$-2 \kappa \hat{n}_{\sigma}^{\lambda \alpha \beta} \Delta_{\alpha \beta}^{\sigma}$. Thus,

$$
\begin{aligned}
\hat{d}_{D K O}^{\lambda} & ={ }_{E} \hat{d}^{\lambda}+{ }_{G B} \hat{d}^{\lambda}=-2 \kappa\left({ }_{E} \hat{n}_{\sigma}{ }^{\lambda \alpha \beta}+{ }_{G B} \hat{n}_{\sigma}{ }^{\lambda \alpha \beta}\right) \Delta_{\alpha \beta}^{\sigma} \\
& =2 \sqrt{-g} \Delta_{\alpha \beta}^{[\alpha} \hat{g}^{\lambda] \beta}+4 \alpha\left(\hat{R}_{\sigma}{ }^{\alpha \beta \lambda}-4 \hat{R}_{\sigma}^{[\alpha} g^{\lambda] \beta}+\delta_{\sigma}^{[\alpha} g^{\lambda] \beta} \hat{R}\right) \Delta_{\alpha \beta}^{\sigma} .
\end{aligned}
$$

The Katz-Livshits [47] approach leads to

$$
\begin{aligned}
\hat{d}_{K L}^{\lambda} & ={ }_{E} \hat{d}^{\lambda}+{ }_{G B} \hat{d}^{\lambda} \\
& =2 \sqrt{-g} \Delta_{\alpha \beta}^{[\alpha} \hat{g}^{\lambda] \beta}+4 \alpha\left(\hat{R}_{\sigma}{ }^{\alpha \beta \lambda}-2 \hat{R}_{\sigma}^{[\alpha} g^{\lambda] \beta}-2 \delta_{\sigma}^{[\alpha} \hat{R}^{\lambda] \beta}+\delta_{\sigma}^{[\alpha} g^{\lambda] \beta} \hat{R}\right) \Delta_{\alpha \beta}^{\sigma} .
\end{aligned}
$$

As is seen, in GR case both of these lead to the choice of KBL. Keeping in mind (7.5) - (3.5), we derive the superpotential in the canonical prescription (2.11) for the EGB gravity:

$$
\begin{aligned}
\hat{\mathcal{I}}_{C}^{\alpha \beta} & ={ }_{E} \hat{\mathcal{I}}_{C}^{\alpha \beta}+{ }_{G B} \hat{\mathcal{I}}_{C}^{\alpha \beta} \\
& =\frac{1}{\kappa}\left(\hat{g}^{\rho[\alpha} \bar{D}_{\rho} \xi^{\beta]}+\hat{g}^{\rho[\alpha} \Delta_{\rho \sigma}^{\beta]} \xi^{\sigma}-\overline{D^{[\alpha} \hat{\xi}^{\beta]}}+\xi^{[\alpha}{ }_{E} \hat{d}^{\beta]}\right) \\
& +{ }_{G B} \hat{\imath}_{C}^{\alpha \beta}-{ }_{G B} \overline{\hat{\imath}_{C}^{\alpha \beta}}+\kappa^{-1} \xi^{[\alpha}{ }_{G B} \hat{d}^{\beta]}
\end{aligned}
$$

where ${ }_{G B} \hat{d}^{\alpha}$ is derived from (3.4) or (3.5). The full Einstein part ${ }_{E} \hat{\mathcal{I}}_{C}^{\alpha \beta}$ is exactly the KBL superpotential both in $4 \mathrm{D}$ GR [13] and in multi-D GR [23, 47].

Now let us turn to the Belinfante corrected superpotential (2.13) in EGB gravity. Substituting the correspondent coefficients (2.10) into (2.12) one obtains

$$
\begin{aligned}
\hat{\imath}_{B}^{\alpha \beta} & =E^{\hat{\imath}_{B}^{\alpha \beta}}+{ }_{G B} \hat{\imath}_{B}^{\alpha \beta} \\
& =\frac{1}{\kappa}\left[\left(\delta_{\sigma}^{[\alpha} \bar{D}_{\lambda} \hat{g}^{\beta] \lambda}-\bar{D}^{[\alpha} \hat{g}^{\beta] \rho} \bar{g}_{\rho \sigma}\right) \xi^{\sigma}+\hat{g}^{\lambda[\alpha} \bar{D}_{\lambda} \xi^{\beta]}\right] \\
& +\frac{\alpha}{\kappa} \bar{D}_{\lambda}\left\{\hat{R}_{\sigma}{ }^{\lambda \alpha \beta}+4 g^{\lambda[\alpha} \hat{R}_{\sigma}^{\beta]}+\left[2 \hat{R}_{\tau}{ }^{\rho \lambda[\alpha}-2 \hat{R}^{\rho \lambda}{ }_{\tau}^{[\alpha}-8 \hat{R}_{\tau}^{\lambda} g^{\rho[\alpha}\right.\right. \\
& \left.\left.+4 \hat{R}_{\tau}^{\rho} g^{\lambda[\alpha}+4 g^{\rho \lambda} \hat{R}_{\tau}^{[\alpha}+2 \hat{R}\left(\delta_{\tau}^{\lambda} g^{\rho[\alpha}-\delta_{\tau}^{\rho} g^{\lambda[\alpha}\right)\right] \bar{g}^{\beta] \tau} \bar{g}_{\rho \sigma}\right\} \xi^{\sigma} \\
& -\frac{2 \alpha}{\kappa}\left\{\hat{R}_{\sigma}^{\lambda \alpha \beta}+4 g^{\lambda[\alpha} \hat{R}_{\sigma}^{\beta]}+\delta_{\sigma}^{[\alpha} g^{\beta] \lambda} \hat{R}\right\} \bar{D}_{\lambda} \xi^{\sigma} .
\end{aligned}
$$

The barred expression is significantly simpler

$$
\begin{aligned}
& \overline{\hat{\imath}_{B}^{\alpha \beta}}={ }_{E} \overline{\hat{\imath}_{B}^{\alpha \beta}}+{ }_{G B} \overline{\hat{\imath}_{B}^{\alpha \beta}} \\
= & \frac{1}{\kappa} \overline{\hat{g}^{\lambda[\alpha} D_{\lambda} \xi^{\beta]}}-\frac{2 \alpha}{\kappa}\left\{\overline{\hat{R}}_{\sigma}^{\lambda \alpha \beta}+4 \overline{g^{\lambda[\alpha} \hat{R}_{\sigma}^{\beta]}}+\delta_{\sigma}^{[\alpha} \overline{g^{\beta] \lambda} \hat{R}}\right\} \bar{D}_{\lambda} \xi^{\sigma} .
\end{aligned}
$$

Thus, keeping in mind (3.7) and (3.8), we derive the superpotential in the Belinfante corrected prescription (2.13) for the EGB gravity:

$$
\begin{aligned}
\hat{\mathcal{I}}_{B}^{\alpha \beta} & ={ }_{E} \hat{\mathcal{I}}_{B}^{\alpha \beta}+{ }_{G B} \hat{\mathcal{I}}_{B}^{\alpha \beta} \\
& =\frac{1}{\kappa}\left(\xi^{[\alpha} \bar{D}_{\lambda} \hat{l}^{\beta] \lambda}-\bar{D}^{[\alpha} \hat{l}_{\sigma}^{\beta]} \xi^{\sigma}+\hat{l}^{\lambda[\alpha} \bar{D}_{\lambda} \xi^{\beta]}\right)+{ }_{G B} \hat{\imath}_{B}^{\alpha \beta}-{ }_{G B} \overline{\hat{\imath}_{B}^{\alpha \beta}}
\end{aligned}
$$

where $\hat{l}^{\alpha \beta}=\hat{g}^{\alpha \beta}-\overline{\hat{g}^{\alpha \beta}}$. The Einstein part ${ }_{E} \hat{\mathcal{I}}_{B}^{\alpha \beta}$, being constructed in arbitrary $D$ dimensions, has exactly the form of the Belinfante corrected superpotential in the 4D GR [16].

At last, turn to the symmetrical superpotential (2.20) in the framework of the EGB case. To concretize a calculation and to have a possibility to compare with [28], we define the perturbations from the set (2.14) as $h^{a}=g_{\alpha \beta}-\bar{g}_{\alpha \beta}=h_{\alpha \beta}$. Thus the Lagrangian in (2.19) has to be calculated as $\hat{\mathcal{L}}_{1}=-(2 \kappa)^{-1} h_{\alpha \beta} \delta \overline{\hat{\mathcal{L}}}_{E G B} / \delta \bar{g}_{\alpha \beta}$. 
Calculating on this basis the coefficients (2.21) one has

$$
\begin{aligned}
& N^{\rho \lambda \mid \mu \nu}=N_{E}^{\rho \lambda \mid \mu \nu}+N_{G B}^{\rho \lambda \mid \mu \nu}= \\
& \text { - } \frac{1}{4 \kappa}\left[\bar{g}^{\rho \lambda} h^{\mu \nu}+\bar{g}^{\mu \nu} h^{\rho \lambda}-\bar{g}^{\rho(\mu} h^{\nu) \lambda}-\bar{g}^{\lambda(\mu} h^{\nu) \rho}+h_{\sigma}^{\sigma}\left(\bar{g}^{\rho(\mu} \bar{g}^{\nu) \lambda}-\bar{g}^{\rho \lambda} \bar{g}^{\mu \nu}\right)\right] \\
& \text { - } \frac{\alpha}{2 \kappa}\left\{h _ { \sigma } ^ { \sigma } \left[\left(\bar{g}^{\rho(\mu} \bar{g}^{\nu) \lambda}-\bar{g}^{\rho \lambda} \bar{g}^{\mu \nu}\right) \bar{R}-2\left(\bar{g}^{\rho(\mu} \bar{R}^{\nu) \lambda}+\bar{R}^{\rho(\mu} \bar{g}^{\nu) \lambda}\right)+2\left(\bar{g}^{\rho \lambda} \bar{R}^{\mu \nu}+\bar{R}^{\rho \lambda} \bar{g}^{\mu \nu}\right)\right.\right. \\
& \left.+2 \bar{R}^{\rho(\mu \nu) \lambda}\right]+\left(\bar{g}^{\rho \lambda} h^{\mu \nu}+\bar{g}^{\mu \nu} h^{\rho \lambda}-\bar{g}^{\rho(\mu} h^{\nu) \lambda}-\bar{g}^{\lambda(\mu} h^{\nu) \rho}\right) \bar{R}+2\left(h^{\rho(\mu} \bar{R}^{\nu) \lambda}+\bar{R}^{\rho(\mu} h^{\nu) \lambda}\right) \\
& -2\left(h^{\rho \lambda} \bar{R}^{\mu \nu}+\bar{R}^{\rho \lambda} h^{\mu \nu}\right)-4\left(\bar{g}^{\rho \lambda} \bar{R}_{\sigma}^{(\mu} h^{\nu) \sigma}+\bar{g}^{\mu \nu} \bar{R}_{\sigma}^{(\rho} h^{\lambda) \sigma}\right)+4\left(\bar{R}_{\sigma}^{(\rho} \bar{g}^{\lambda)(\mu} h^{\nu) \sigma}+\bar{R}_{\sigma}^{(\mu} \bar{g}^{\nu)(\rho} h^{\lambda) \sigma}\right) \\
& \text { - } 2\left(\bar{g}^{\rho(\mu} \bar{g}^{\nu) \lambda}-\bar{g}^{\rho \lambda} \bar{g}^{\mu \nu}\right) \bar{R}_{\sigma}^{\tau} h_{\tau}^{\sigma}-4\left(\bar{R}_{\sigma}{ }^{(\rho \lambda)(\mu} h^{\nu) \sigma}+\bar{R}_{\sigma}^{(\mu \nu)(\rho} h^{\lambda) \sigma}\right) \\
& \left.+2 h_{\sigma \tau}\left(\bar{R}^{\sigma \mu \tau(\rho} \bar{g}^{\lambda) \nu}+\bar{R}^{\sigma \nu \tau(\rho} \bar{g}^{\lambda) \mu}\right)+2 h_{\sigma \tau}\left(\bar{g}^{\rho \lambda} \bar{R}^{\sigma \mu \nu \tau}+\bar{g}^{\mu \nu} \bar{R}^{\sigma \rho \lambda \tau}\right)\right\} .
\end{aligned}
$$

Substituting them into (2.20) one obtains

$$
\begin{aligned}
\hat{\mathcal{I}}_{S}^{\alpha \beta} & ={ }_{E} \hat{\mathcal{I}}_{S}^{\alpha \beta}+{ }_{G B} \hat{\mathcal{I}}_{S}^{\alpha \beta} \\
& =\frac{\sqrt{-\bar{g}}}{\kappa}\left(\xi_{\nu} \bar{D}^{[\alpha} h^{\beta] \nu}-\xi^{[\alpha} \bar{D}_{\nu} h^{\beta] \nu}+\xi^{[\alpha} \bar{D}^{\beta]} h-h^{\nu[\alpha} \bar{D}_{\nu} \xi^{\beta]}+\frac{1}{2} h \bar{D}^{[\alpha} \xi^{\beta]}\right) \\
& +\frac{4}{3}\left(2 \xi_{\sigma} \bar{D}_{\lambda} \hat{N}_{G B}^{\sigma[\alpha] \beta] \lambda}-\hat{N}_{G B}^{\sigma[\alpha \mid \beta] \lambda} \bar{D}_{\lambda} \xi_{\sigma}\right) .
\end{aligned}
$$

This expression coincides with the Deser-Tekin superpotential [28] if one chooses the EGB gravity on the AdS background. The expression (3.11) calculated for $h^{a}=h_{\alpha \beta}$ is a particular case of the more general case with the decomposition (2.14) and the redefinition (2.18):

$$
\begin{aligned}
\hat{\mathcal{I}}_{S}^{\alpha \beta} & ={ }_{E} \hat{\mathcal{I}}_{S}^{\alpha \beta}+{ }_{G B} \hat{\mathcal{I}}_{S}^{\alpha \beta}=\frac{1}{\kappa}\left(\hat{l}_{(a)}^{\sigma[\mu} \bar{D}_{\sigma} \xi^{\rho]}+\xi^{[\mu} \bar{D}_{\sigma} \hat{l}_{(a)}^{\rho] \sigma}-\bar{D}^{[\mu} \hat{l}_{(a) \sigma}^{\rho]} \xi^{\sigma}\right) \\
& +\frac{4}{3}\left(2 \xi_{\sigma} \bar{D}_{\lambda} \hat{N}_{G B}^{\sigma[\alpha \mid \beta] \lambda}\left(\hat{l}_{(a)}\right)-\hat{N}_{G B}^{\sigma[\alpha \mid \beta] \lambda} \bar{D}_{\lambda}\left(\hat{l}_{(a)}\right) \xi_{\sigma}\right) .
\end{aligned}
$$

Of course, the above defined superpotentials differs one from another. However, returning to 4D GR, as we remarked in Introduction, all the three approaches satisfy the main tests. Indeed, calculating at infinity the surface integrals (2.4) for isolated systems in 4D GR [22], one obtains the same accepted results with using each of the Einstein parts in the superpotentials (3.6), (3.9) and (3.12). In the case of asymptotically flat spacetimes at spatial infinity the situation is more simple: all the differences between these kinds of superpotentials do not contribute into (2.4) [16, 22]. Analogously, in the next sections, we show that all the three approaches give the standard mass for the S-AdS BH in EGB gravity.

A more complicated case is an isolated system at null infinity. In 4D GR both the canonical and Belinfante corrected approaches give the same result [16] coinciding with the standard Bondi-Sachs energy-momentum flux [49]. Another situation is in the symmetrical approach. A different choice of variables from (2.2) and respectively a different decompositions (2.14) lead to different $h^{a}$. Then variables $\hat{l}_{(a)}^{\mu \nu}$ in (2.18) differ one from other in the second order in perturbations. This difference is explicitly incorporated into the left hand side of (2.16), and respectively into the superpotentials (2.20), or (3.12) in the EGB case. Already in [49] it has been remarked that the difference in the second order is important in a calculation for a radiating isolated system in 4D GR. It turns out [22] that only the choice $h^{a}=\hat{g}^{\mu \nu}-\overline{\hat{g}}^{\mu \nu} \equiv \hat{l}^{\mu \nu}$ gives the standard result [49] (not another, including $h^{a}=h_{\alpha \beta}$ ). Concerning EGB gravity, a) the different $\hat{l}_{(a)}^{\mu \nu}$ in $(2.18)$ give the same standard mass for the S-AdS BH calculated below, and b) we only plan to consider radiating systems in future.

Considering different variables $\hat{l}_{(a)}^{\mu \nu}$ in (2.18), we note also that only for $h^{a}=\hat{l}^{\alpha \beta}$ the Einstein parts in (3.9) and (3.12) coincide: ${ }_{E} \hat{\mathcal{I}}_{B}^{\mu \nu}={ }_{E} \hat{\mathcal{I}}_{S}^{\mu \nu}$ that could be interpreted as an advantage of the choice $h^{a}=\hat{l}^{\alpha \beta}$. 
However, in an arbitrary gravitation theory $\hat{\mathcal{I}}_{B}^{\mu \nu} \neq \hat{\mathcal{I}}_{S}^{\mu \nu}$, compare the Gauss-Bonnet parts in (3.9) and (3.12). Nevertheless, because many multi-D gravities, as a rule, are the Einstein theories with corrections, one again could prefer $\hat{l}^{\mu \nu}$ from the set $\hat{l}_{(a)}^{\mu \nu}$ comparing the Einstein parts only.

\section{Static spherically symmetric solutions}

Many interesting solutions in the vacuum EGB gravity have the Schwarzschild-like form:

$$
d s^{2}=-f d t^{2}+f^{-1} d r^{2}+r^{2} \sum_{a, b}^{D-2} q_{a b} d x^{a} d x^{b}
$$

where $f=f(r)$, the last term describes $(D-2)$-dimensional sphere of the radius $r$, and $q_{a b}$ depends on coordinates on the sphere only. As a background we choose again the solution of the same form:

$$
d \bar{s}^{2}=-\bar{f} d t^{2}+(\bar{f})^{-1} d r^{2}+r^{2} \sum_{a, b}^{D-2} q_{a b} d x^{a} d x^{b} .
$$

In this section, we present the general formulae, which can be used for calculating the mass of the perturbed system (4.1) with respect to the background (4.2). Due to a spherical symmetry, for calculating conserved quantities one needs only in the 01-component of superpotentials in (2.4). We also note that a background of the type (4.2) has the Killing vector $\lambda^{\alpha}=\{-1, \mathbf{0}\}$, which is just necessary for calculating a mass of the system.

At first, we calculate the component $\hat{\mathcal{I}}_{C}^{01}$ of the canonical superpotential (3.6). We use the metrics (4.1) and (4.2) directly in (7.5) and (3.4) with $\xi^{\alpha}=\lambda^{\alpha}$. Thus,

$$
\begin{aligned}
\hat{\imath}_{C}^{01} & +\kappa^{-1} \lambda^{[0} \hat{d}_{D K O}^{1]}=\frac{\sqrt{-\bar{g}}}{2 \kappa r}\left[\frac{r \bar{f}^{\prime}}{2}\left(\frac{f}{\bar{f}}+\frac{\bar{f}}{f}\right)-(f-\bar{f})(D-2)\right] \\
& -\frac{\alpha \sqrt{-\bar{g}}}{\kappa r^{2}}(D-2)\left[f\left(f^{\prime}-r f^{\prime \prime}\right)-\bar{f} f^{\prime}(D-3)\right] \\
& -\frac{\alpha \sqrt{-\bar{g}}}{\kappa r^{3}}(D-2)(D-3)(f-1)\left[\frac{r \bar{f}^{\prime}}{2}\left(\frac{f}{\bar{f}}+\frac{\bar{f}}{f}\right)-(f-\bar{f})(D-2)+2 \bar{f}\right] .
\end{aligned}
$$

We have used $\sqrt{-g}=\sqrt{-\bar{g}}$. The 01-component calculated with the divergence (3.5) is

$$
\begin{aligned}
\hat{\imath}_{C}^{01} & +\kappa^{-1} \lambda^{[0} \hat{d}_{K L}^{1]}=\frac{\sqrt{-\bar{g}}}{2 \kappa r}\left[\frac{r \bar{f}^{\prime}}{2}\left(\frac{f}{\bar{f}}+\frac{\bar{f}}{f}\right)-(f-\bar{f})(D-2)\right] \\
& -\frac{\alpha \sqrt{-\bar{g}}}{\kappa r^{2}}(D-2)\left[\bar{f}\left(f^{\prime}-r f^{\prime \prime}\right)+(f-2 \bar{f}) f^{\prime}(D-3)\right] \\
& -\frac{\alpha \sqrt{-\bar{g}}}{\kappa r^{3}}(D-2)(D-3)(f-1)\left[\frac{r \bar{f}^{\prime}}{2}\left(\frac{f}{\bar{f}}+\frac{\bar{f}}{f}\right)-(f-\bar{f}) D+2 \bar{f}\right] .
\end{aligned}
$$

The background expression for both the cases (7.7) and (7.8) is the unique one

$$
\begin{aligned}
\overline{\hat{\imath}_{C}^{01}} & =\frac{\sqrt{-\bar{g}}}{2 \kappa} \bar{f}^{\prime}+\frac{\alpha \sqrt{-\bar{g}}}{\kappa r^{2}}(D-2) \bar{f}\left[r \bar{f}^{\prime \prime}+\bar{f}^{\prime}(D-4)\right] \\
& -\frac{\alpha \sqrt{-\bar{g}}}{\kappa r^{3}}(D-2)(D-3)(\bar{f}-1)\left(r \bar{f}^{\prime}+2 \bar{f}\right) .
\end{aligned}
$$

Of course, it is also calculated directly from (7.6). The component $\hat{\mathcal{I}}_{C}^{01}$ in (3.6) is obtained after substraction of (7.9) from (7.7) or (7.8). 
Now, calculate the component $\hat{\mathcal{I}}_{B}^{01}$ of the Belinfante corrected superpotential (3.9) for the relative systems (4.1) and (4.2) and the displacement vector $\xi^{\alpha}=\lambda^{\alpha}$. For (3.7) one has

$$
\begin{aligned}
\hat{\imath}_{B}^{01} & =\frac{\sqrt{-\bar{g}}}{2 \kappa r}\left[\frac{r \bar{f}^{\prime}}{2}\left(3 \frac{f}{\bar{f}}-\frac{\bar{f}}{f}\right)-r f^{\prime}\left(1-\frac{\bar{f}^{2}}{f^{2}}\right)-(f-\bar{f})(D-2)\right] \\
& -\frac{\alpha \sqrt{-\bar{g}}}{\kappa r^{2}}(D-2)\left[\bar{f}\left(f^{\prime}-r f^{\prime \prime}\right)\left(1-\frac{\bar{f}}{f}\right)-f f^{\prime}\left(1-\frac{\bar{f}^{2}}{f^{2}}\right)(D-3)\right] \\
& +\frac{\alpha \sqrt{-\bar{g}}}{\kappa r^{3}}(f-1)(D-2)(D-3)\left[\frac{r \bar{f}^{\prime}}{2}\left(\frac{\bar{f}}{f}-3 \frac{f}{\bar{f}}\right)+\left(r f^{\prime}-2 f\right)\left(1-\frac{\bar{f}^{2}}{f^{2}}\right)\right. \\
& +(f-\bar{f})(D-2)] .
\end{aligned}
$$

The barred expression (4.6) is

$$
\overline{\hat{\imath}_{B}^{01}}=\frac{\sqrt{-\bar{g}}}{2 \kappa} \bar{f}^{\prime}-\frac{\alpha \sqrt{-\bar{g}}}{\kappa r^{2}}(D-2)(D-3) \bar{f}^{\prime}(\bar{f}-1) .
$$

Of course, it is also calculated directly from (3.8). The component $\hat{\mathcal{I}}_{B}^{01}$ in (3.9) is obtained after subtraction of (4.7) from (4.6).

At last, we calculate the 01-component of the symmetrical superpotential. For the metric perturbations of (4.1) with respect to (4.2) one has only the non-zero components $h_{00}=-(f-\bar{f}), \quad h_{11}=-(f-\bar{f}) / f \bar{f}$. Using them, $\xi^{\alpha}=\lambda^{\alpha}$ and (4.2) in (3.11) directly one obtains

$$
\hat{\mathcal{I}}_{S}^{01}=\frac{\sqrt{-\bar{g}}}{2 \kappa r}(D-2)(f-\bar{f}) \frac{\bar{f}}{f}\left[-1+2 \alpha(\bar{f}-1) \frac{(D-3)(D-4)}{r^{2}}\right] .
$$

This expression is significantly simpler than corresponding expressions in the other approaches. Besides, being proportional to $f-\bar{f}$, it directly describes the perturbed system. At last, we note that all the final expressions of this section are exact (not approximate).

\section{The mass of the Schwarzschild-AdS black hole}

As an example of (4.1), we consider the S-AdS solution [44]:

$$
f(r)=1-\frac{r^{2} \Lambda^{\prime}}{(D-2)(D-1)}\left\{1 \pm \sqrt{1-\frac{4 \Lambda_{0}}{\Lambda^{\prime}}-\frac{2(D-2)(D-1)}{\Lambda^{\prime}} \frac{\mu}{r^{D-1}}}\right\}
$$

where $\mu$ is a constant of integration, and $\Lambda^{\prime}=-(D-2)(D-1) / 2 \alpha(D-4)(D-3)$ is defined only by the Gauss-Bonnet term. For the sake of simplicity (to exclude numerous nuances) we restrict ourselves to $D \geq 5$. As the important backgrounds we consider the AdS spacetime. It is defined by (5.1) at $\mu=0$ with the effective cosmological constant $\Lambda_{\text {eff }}$ :

$$
\bar{f}(r)=1-r^{2} \frac{2 \Lambda_{e f f}}{(D-1)(D-2)} ; \quad \Lambda_{e f f}=\frac{\Lambda^{\prime}}{2}\left(1 \pm \sqrt{1-\frac{4 \Lambda_{0}}{\Lambda^{\prime}}}\right) .
$$

In the linear approximation the perturbation (5.1) with respect to (5.2) is

$$
\Delta f=f(r)-\bar{f}(r)= \pm\left(\sqrt{1-\frac{4 \Lambda_{0}}{\Lambda^{\prime}}}\right)^{-1} \frac{\mu}{r^{D-3}} .
$$

We assume $1-4 \Lambda_{0} / \Lambda^{\prime} \neq 0$, the situation $1-4 \Lambda_{0} / \Lambda^{\prime}=0$ is discussed in the last section. 
With a desire to consider the solution (5.1) as a black hole solution one has to choose the "-" sign (lower sign) because only then one can define a horizon radius $r_{+}$of the black hole setting $f=0$. Besides, there is a qualitative difference between the cases $D \geq 6$ and $D=5$. In the first case, vanishing the constant of integration $\mu \rightarrow 0$ corresponds vanishing the horizon $r_{+} \rightarrow 0$. Thus, $\mu$ readily can be interpreted as a mass parameter $M=\mu$, and the AdS solution (5.2) can be interpreted as a natural background for such black holes. Then the asymptotic perturbations (5.3) look quite natural. In the case $D=5$, the situation is different: the horizon $r_{+} \rightarrow 0$ vanishes if $\mu \rightarrow \mu_{0}$ where $\mu_{0}=\alpha(D-3)(D-4)=2 \alpha=-6 / \Lambda^{\prime}$. Then one can define a mass parameter as $M=\mu-\mu_{0}$, for which again $r_{+} \rightarrow 0$ at $M \rightarrow 0$. Thus for such a black hole in 5 dimensions it is natural to choose a vacuum background at $M=0$ in (5.1):

$$
\bar{f}(r)=1-\frac{r^{2} \Lambda^{\prime}}{12}\left\{1-\sqrt{1-\frac{4 \Lambda_{0}}{\Lambda^{\prime}}+\left(\frac{12}{r^{2} \Lambda^{\prime}}\right)^{2}}\right\} .
$$

Only for negative $M=-\mu_{0}$ one approaches the AdS background with $\bar{f}$ in (5.2), $\mu_{0}$ is called as a gap between the AdS spacetime and a real black hole vacuum [4]. Then, for $D=5$, the perturbation with respect to (5.4) asymptotically is

$$
\Delta f(r)=f(r)-\bar{f}(r)=-\frac{M}{r^{2}}\left(\sqrt{1-\frac{4 \Lambda_{0}}{\Lambda^{\prime}}}\right)^{-1}\left[1+\frac{6}{r^{4} \Lambda^{\prime}}\left(M-\frac{12}{\Lambda^{\prime}}\right)\right] .
$$

Here, the main order coincides with the order of a difference between (5.4) and (5.2), therefore we conserve the next order.

To calculate the mass of the S-AdS BH (5.1) we use the integral (2.4) under the requirement $r \rightarrow$ $\infty$. At first we turn to the canonical prescription for both the cases (7.7) and (7.8). We consider the linear approximation of $\hat{\mathcal{I}}_{C}^{01}$ both in the perturbation $\Delta f$ in (5.3) with respect to (5.2), $D \geq 6$, and in the perturbation $\Delta f$ in (5.5) with respect to $(5.4), D=5$. The unique expression can be derived in all the cases because only the main orders in (5.2) and (5.3), or in (5.4) and (5.5), contribute into the surface integral (2.4). The asymptotic relations $(\Delta f)^{\prime}=-(D-3) \Delta f / r, \quad(\bar{f})^{\prime}=2 \bar{f} / r$ are used. Then the linear expression is

$$
\hat{\mathcal{I}}_{C}^{01}=-\frac{\sqrt{-\bar{g}}}{2 \kappa r} \Delta f(D-2)+\frac{\alpha \sqrt{-\bar{g}}}{\kappa r^{3}} \Delta f \bar{f}(D-2)(D-3)(D-4) .
$$

Substituting the main order from (5.2) or (5.4) we obtain

$$
\hat{\mathcal{I}}_{C}^{01}=-\frac{\sqrt{-\bar{g}}}{2 \kappa r} \Delta f(D-2) \sqrt{1-\frac{4 \Lambda_{0}}{\Lambda_{E G B}}} .
$$

Thus, for both the cases (5.3) and (5.5) we have finally in the canonical approach:

$$
\hat{\mathcal{I}}_{C}^{01}=\frac{\sqrt{-\bar{g}}}{2 \kappa r} \frac{M}{r^{D-3}}(D-2) .
$$

Substituting it into (2.4) together with $\sqrt{-\bar{g}}=r^{D-2} \sqrt{\operatorname{det} q_{i j}}=r^{D-2} \Omega_{D-2}$ one gets:

$$
E=(D-2) \frac{M}{4 G_{D}}
$$

that is the accepted result obtained with using the various approaches (see [2] - [9, 23, 28] and references therein). Recall also that both the cases (7.7) and (7.8) lead to (5.9). Formally the expression (5.7) holds for $D \geq 5$. Thus, for $D=5$ on AdS background one can change $M \rightarrow M+\mu_{0}$ in (5.8) and, respectively in (5.9). This result could be interpreted as an energy of the system presented by the BH together with the 
"mass gap" $\mu_{0}$ on the AdS background. Then, indeed, one has to calculate the energy of the 5D BH in the vacuum "mass gap".

To calculate the mass of the S-AdS BH (5.1) with the use of the Belinfante corrected expressions (4.6) and (4.7) we again consider the linear approximation and follow all the same steps of the previous derivation. It turns out that all the formulae and conclusions of the canonical prescription from (5.6) are repeated exactly.

In the framework of the field-theoretical approach it is enough the linear approximation: $h_{00}=h^{11}=$ $-\Delta f$ where $\Delta f$ can be defined both in (5.3) and in (5.5). To calculate the mass of the S-AdS BH (5.1) we consider linear approximation of (4.8) and follow all the same steps of the previous approaches. Again, it turns out that all the formulae and comments from (5.6) are repeated exactly.

At the above consideration, to stress an importance of the notion of the "mass gap" in 5 dimensions and to show clearly a calculation on the background of the "mass gap" vacuum, we are restricting to the "minus" branch in (5.1). However, the naive interpretation of the mass permits to consider both the branches in (5.1) with the next conserving the two signs from (5.2) to (5.5), like in $[7,29,31]$. One obtains again the accepted quantity (5.9).

\section{Discussion and concluding remarks}

There are infinitely many possibilities to construct conserved quantities in metric theories. In the recent work [50] by Pitts the very interesting idea is suggested, where instead of many complexes he considers any infinitecomponent object that is conserved and that makes sense in every coordinate system/gauge. This idea is in a convenience with our principal position, when all the known possibilities to define conserved quantities are important, if they are evidently non-contradictive and satisfy all the acceptable tests. Therefore we consider three approaches simultaneously.

Here, we propagandize the derivation of perturbations in the prescription of a field theory in the classical form. Considering a background as an arena for perturbations, we connect conserved charges with its symmetries expressed by Killing vectors. The symmetrical approach is more illustrative because it derives perturbations on backgrounds explicitly. The AdS background is more popular therefore it is useful to consider just this case. We substitute the metric (5.2) into $N_{G B}^{\rho \lambda \mid \mu \nu}$ in (3.10) and obtain the relation: $N_{G B}^{\rho \lambda \mid \mu \nu}=$ $-\left(1 \pm \sqrt{1-4 \Lambda_{0} / \Lambda^{\prime}}\right) N_{E}^{\rho \lambda \mid \mu \nu}$. After that the symmetrical superpotential (2.20) in the EGB gravity (3.11) for of arbitrary type perturbations $h_{\mu \nu}$ on the AdS background and with arbitrary $\xi^{\alpha}$ acquires the form [31]:

$$
\hat{\mathcal{I}}_{S}^{\mu \rho} \equiv \pm \frac{\sqrt{-\bar{g}}}{\kappa} \sqrt{1-\frac{4 \Lambda_{0}}{\Lambda^{\prime}}}\left(\xi^{[\mu} \bar{D}_{\nu} h^{\rho] \nu}-\xi_{\nu} \bar{D}^{[\mu} h^{\rho] \nu}-\xi^{[\mu} \bar{D}^{\rho]} h-h^{\nu[\mu} \bar{D}^{\rho]} \xi_{\nu}-\frac{1}{2} h \bar{D}^{[\mu} \xi^{\rho]}\right) .
$$

If the Killing vectors are used, then a) it is expressed through the Abbott-Deser superpotential in the Einstein theory [19, 28], $\hat{I}_{A D}^{\mu \rho}$, as $\hat{\mathcal{I}}_{S}^{\mu \rho}=\mp \sqrt{1-4 \Lambda_{0} / \Lambda^{\prime}} \hat{I}_{A D}^{\mu \rho}$; b) the same expression (6.1), note the signs, has been approached in $[7,29]$, where the Deser-Tekin results [28] were developed. Keeping in mind the generalized metric perturbations (2.18), the simplification of (3.12) to the AdS background gives

$$
\hat{\mathcal{I}}_{S}^{\mu \rho} \equiv \frac{1}{\kappa}\left[1_{E}-\left(1 \pm \sqrt{1-\frac{4 \Lambda_{0}}{\Lambda^{\prime}}}\right)_{G B}\right]\left(\hat{l}_{(a)}^{\sigma[\mu} \bar{D}_{\sigma} \xi^{\rho]}+\xi^{[\mu} \bar{D}_{\sigma} \hat{l}_{(a)}^{\rho] \sigma}-\bar{D}^{[\mu} \hat{l}_{(a) \sigma}^{\rho]} \xi^{\sigma}\right) .
$$

Thus, the superpotential (6.1) is the one of the set (6.2). The Einstein part in (6.2) formally coincides with the one in $4 \mathrm{D}$ GR $[16,22]$.

The superpotential (3.11) can be thought as a generalization of (6.1). Padilla, restricting his own general results [7] to the AdS asymptotic, has found out a consistence with the Deser-Tekin result (6.1) also. We 
see that on the level of the AdS background there is the consistence between the now discussed results: of the present paper, Deser-Tekin's and Padilla's. Returning to the three approaches presented here, we have demonstrated that they are powerful for calculations not only on AdS backgrounds, but also on not maximally symmetrical backgrounds, like (5.4). Thus, concerning the criticism of the Deser and Tekin approach in [7], we show that developing their method we are not restricted only by the AdS background. But, then it is interesting and important to compare the presented here general approaches with the general Padilla approach [7]. Of course, they are different: at least, our methods are Lagrangian and are based on the conservation laws of the type (2.3), whereas in [7] surface integrals in the Hamiltonian derivation play a main role. However, Padilla applying his method, give the formula (129) in [7] for calculating the mass of the model that we present in section 4: the perturbed system of the type (4.1) is considered with respect to the background with the metric (4.2). His integrand in our notations could be rewritten as

$$
\hat{\mathcal{I}}^{01}=-\frac{\sqrt{-\bar{g}}}{2 \kappa r} \sqrt{f}\left\{6(\sqrt{f}-\sqrt{\bar{f}})+\frac{24 \alpha}{r^{2}}\left[(\sqrt{f}-\sqrt{\bar{f}})-\frac{1}{3}(f \sqrt{f}-\bar{f} \sqrt{\bar{f}})\right]\right\}
$$

which is quite different from each of the correspondent expressions in our approaches (7.7) - (4.8). Nevertheless, substituting the perturbations (5.5) on the background (5.4) into (6.3) one obtains again the accepted result (5.9) with $D=5$. Thus one can conclude that the presented here methods are consistent with the Padilla approach on the more complicated backgrounds than the AdS one.

The results of section 5 have been obtained under the assumption that the factor $\sqrt{1-4 \Lambda_{0} / \Lambda^{\prime}}$ is not equal to zero. Indeed, at the linear approximations the 01-component of the superpotentials for calculating the mass in all the three approaches is proportional to $\sqrt{1-4 \Lambda_{0} / \Lambda^{\prime}}$ (see $(5.7)$ ). Thus, for $\Lambda^{\prime}=4 \Lambda_{0}$ the global mass, probably, has to be treated as vanishing!? This fact is remarked in the works [4, 7, 25, 29], however without detailed discussion. Deruelle and Morisava [25] (canonical derivation), and Deser, Kanik and Tekin [29] (field-theoretical derivation) have found out that not only mass, but also angular momentum expressions for the Kerr-AdS solution in EGB gravity have the same coefficient.

Considering the condition $\Lambda^{\prime}=4 \Lambda_{0}$ authors, as a rule, send readers to the paper [51], where the situation is explained that gravitons do not propagate on AdS backgrounds. Indeed, the linearized EGB vacuum equations calculated as the left hand side in the equations (2.16) on the AdS background has the same factor and disappear at the condition $\Lambda^{\prime}=4 \Lambda_{0}$. However, only in the framework of the field-theoretical approach there is a direct connection between vanishing the linear equations and the superpotentials (see (6.2)). Inversely, in the canonical and the Belinfante corrected derivations such a fact does not has a place: the expressions (3.6) and (3.9) linearized around the AdS background in arbitrary perturbations do not proportional to $\sqrt{1-4 \Lambda_{0} / \Lambda^{\prime}}$. The field-theoretical interpretation of the condition $\Lambda^{\prime}=4 \Lambda_{0}$ looks also as a more suitable. Indeed, vanishing the linear left hand side in (2.16) leads to vanishing the total energymomentum at the right hand side. For the case, when the matter is absent, this means that the whole energy-momentum of the pure metric perturbations is to be equal to zero. It is absence of gravitons!

Difficulties connected with the condition $\Lambda^{\prime}=4 \Lambda_{0}$ appear even if the linear approximation does not disappear. Thus, if $\Lambda^{\prime}=4 \Lambda_{0}$ our approaches do not give acceptable results on the background of the "mass gap" in 5 dimensions. Therefore, in future it is important to connect presented here approaches with methods where the mass in this degenerated case is defined acceptably; they are, e.g., the Regge and Teitelboim method [52] in multi- $D$ application (see, e.g., [2]) and the Paddila [7] Hamiltonian constructions.

Keeping in mind a future development we note that the static spherically symmetric Schwarzschildlike solutions could be examined very effectively. However, the presented formalism is more universal. Its methods can be especially transparent also for the cases both of rotating black hole solutions (see, e.g., [33] 
- [39]) and of radiating solutions (see, e.g., [40] - [42]). It can be also developed in the framework of the Lovelock theory of the general type [32]; we plan to do this comparing with known results (see. e.g., [8, 47]).

\section{Acknowledgments}

The author is very grateful to Joseph Katz for the prolonged and fruitful discussions, due to which many points have been clarified and the presentation has been significantly improved. The author expresses also his gratitude to Alikram Aliev for deep explanations of his works, very useful conversations and recommendations for future development of the results. The work is supported by the grant No. 09-02-01315-a of the Russian Foundation for Basic Research.

\section{References}

[1] Rubakov V A 2001 Large and infinite extra dimensions Phys. Usp. 44871 [2001 Usp. Fiz. Nauk 171 913] (Preprint hep-ph/0104152)

[2] Crisóstomo J, Troncoso R and Zanelli J 2000 Black hole scan Phys. Rev. D 62084013 (Preprint hep-th/0003271)

[3] Nojiri S, Odintsov S D and Ogushi S 2002 Cosmological and black hole brane-world Universes in higher derivative gravity Phys. Rev. D 65023521 (Preprint hep-th/0108172)

[4] Cai R-G 2002 Gauss-Bonnet black holes in AdS spaces Phys. Rev. D 65084014 (Preprint hepth/01092133)

[5] Emparan R and Reall H S, Black holes in higher dimensions (Preprint arXiv:0801.3471 [hep-th])

[6] Obers N A, Black holes in higher-dimensional gravity (Preprint arXiv:0802.0519 [hep-th])

[7] Paddila A 2003 Surface terms and Gauss-Bonnet Hamiltonian Class. Quantum Grav. 203129 (Preprint gr-qc/0303082)

[8] Allemandi G, Francaviglia M and Raiteri M 2003 Charges and energy in Chern-Simons theories and Lovelock gravity Class. Quantum Grav. 205103 (Preprint gr-qc/0308019)

[9] Okuyama N and Koga J-I 2005 Asymptotically anti-de Sitter spacetimes and conserved quantities in higher curvature gravitational theories Phys. Rev. D 71084009 (Preprint hep-th/0501044)

[10] Kofinas G and Olea R 2006 Vacuum energy in Einstein-Gauss-Bonnet AdS gravity Phys. Rev. D 74 084035 (Preprint hep-th/0606253)

[11] Einstein A 1916 The Hamiltonian principle and general theory of relativity Sitzungsber. preuss. Akad. Wiss. 21111

[12] Von Freud Ph 1939 Über die ausdrücke der gesamtenergie und des gesamtimpulses eins materiellen systems in der allgemeinen relativitätstheorie Ann. of Math. 40417

[13] Katz J, Bičák J and Lynden-Bell D 1997 Relativistic conservation laws and integral constraints for large cosmological perturbations Phys. Rev. D 555957 (Preprint gr-qc/0504041) 
[14] Belinfante F J 1939 On the spin angular momentum and mesons Physica 6887

[15] Papapetrou A 1948 Einstein's theory of gravitation and flat space Proc. R. Irish Ac. 5211

[16] Petrov A N and Katz J 2002 Conserved currents, superpotentials and cosmological perturbations Proc. R. Soc. A, London 458319 (Preprint gr-qc/9911025)

[17] Weinberg S 1972 Gravitation and Cosmology (Wiley, New York)

[18] Deser S 1970 Self-interaction and gauge invariance Gen. Relat. Grav. 19 (Preprint gr-qc/0411023)

[19] Abbott L F and Deser S 1982 Stability of gravity with a cosmological constant Nucl. Phys. B 19576

[20] Grishchuk L P, Petrov A N and Popova A D 1984 Exact theory of the (Einstein) gravitational field in an arbitrary background space-time Commun. Math. Phys. 94379

[21] Petrov A N 2004 Perturbations in the Einstein theory of gravity: Conserved currents Mosc. Univ. Phys. Bull. 59 No 124 [2004 Vestnik Mosk. Univ. Fiz. Astron. No 1 18] (Preprint gr-qc/0402090)

[22] Petrov A N 2008 Nonlinear perturbations and conservation laws on curved backgrounds in GR and other metric theories, 2-nd chapter in the book: Classical and Quantum Gravity Research Eds: Christiansen M N and Rasmussen T K (Nova Science Publishers, N.Y.) 79 (Preprint arXiv:0705.0019 [gr-qc])

[23] Deruelle N, Katz J and Ogushi S 2004 Conserved charges in Einstein-Gauss-Bonnet theory Class. Quantum Grav. 211971 (Preprint gr-qc/0310098)

[24] Deruelle N and Katz J 2005 On the mass of a Kerr-anti-de Sitter spacetime in D dimensions Class. Quantum Grav. 22421 (Preprint gr-qc/0411035)

[25] Deruelle N and Morisawa Y 2005 Mass and angular momenta of Kerr anti-de Sitter spacetimes in Einstein-Gauss-Bonnet theory Class. Quantum Grav. 22933 (Preprint gr-qc/0411135)

[26] Petrov A N 2004 Conserved currents in D-dimensional gravity and brane cosmology Mosc. Univ. Phys. Bull. 59 No 211 [2004 Vestnik Mosk. Univ. Fiz. Astron. No 2 10] (Preprint gr-qc/0401085)

[27] Popova A D and Petrov A N 1988 The dynamic theories on a fixed background in gravitation Int. J. Mod. Phys. A 32651

[28] Deser S and Tekin B 2003 Energy in generic higher curvature gravity theories Phys. Rev. D 67084009 (Preprint hep-th/0212292)

[29] Deser S, Kanik I and Tekin B 2005 Conserved charges in higher D Kerr-AdS spacetimes Class. Quantum Grav. 223383 (Preprint gr-qc/0506057)

[30] Deser S and Tekin B 2007 New energy definition for higher curvature gravities Phys. Rev. D 75103501 (Preprint gr-qc/0701140)

[31] Petrov A N 2005 A note on the Deser-Tekin charges Class. Quantum Grav. 22 L83 (Preprint grqc/0504058)

[32] Lovelock D 1971 The Einstein tensor and its generalizations J. Math. Phys. 12498 
[33] Aliev A N 2006 A slowly rotating charged black hole in five dimensions Mod. Phys. Lett. A 21751 (Preprint gr-qc/0505003)

[34] Aliev A N 2007 Electromagnetic properties of Kerr-anti-de Sitter black holes Phys. Rev. D 75084041 (Preprint hep-th/0702129)

[35] Alexeyev S, Popov N, Startseva M, Barrau A and Grain J 2008 Kerr-Gauss-Bonnet black holes: Exact analitical solution JETP 106710 (Preprint arXiv:0712.3546 [gr-qc])

[36] Bihayy Y and Radu E, Five-dimensional rotating black holes in Einstein-Gauss-Bonnet theory (Preprint arXiv:0801.1021 [hep-th])

[37] Anabalon A, Deruelle N, Morisawa Y, Oliva J, Sasaki M, Tempo D and Troncoso R, Kerr-Schild ansatz in Einstein-Gauss-Bonnet gravity: An exact vacuum solution in five dimensions (Preprint arXiv:0812.3194 [hep-th])

[38] Dehghani M N 2003 Charged rotating black branes in anti-de Sitter Einstein-Gauss-Bonnet gravity Phys. Rev. D 67064017 (Preprint hep-th/0211191)

[39] Aliev A N and Gumrukcuoglu A E 2005 Charged rotating black holes on a 3-brane Phys. Rev. D 71 104027 (Preprint hep-th/0502223)

[40] Ghosh S C and Dawood A K 2008 Radiating black hole solutions in arbitrary dimensions Gen. Relat. Grav. 409 (Preprint arXiv:0801.4342 [gr-qc])

[41] Maeda H and Dadhich N 2007 Matter without matter: novel Kaluza-Klein spacetime in EinsteinGauss-Bonnet gravity Phys. Rev. D $\mathbf{7 5} 044007$ (Preprint hep-th/0611188)

[42] Molina A and Dadhich N, Kaluza-Klein spacetime in Einstein-Gauss-Bonnet gravity (Preprint arXiv:0804.1194 [gr-qc])

[43] Dotti G, Oliva J and Troncoso R, Vacuum solutions with nontrivial boundaries for the Einstein-GaussBonnet theory (Preprint arXiv:0809.4378 [hep-th])

[44] Boulware D C and Deser S 1985 String-generated gravity models Phys. Rev. Lett. 552656

[45] Chang C-C, Nester J M and Chen C-M 1999 Pseudotensors and quasilocal energy-momentum Phys. Rev. Lett. 831897 (Preprint gr-qc/9809040)

[46] Silva S 1999 On superpotentials and charge algebras of gauge theories Nucl. Phys. B 558391 (Preprint hep-th/9809109)

[47] Katz J and Livshits G I 2008 Superpotentials from variational derivatives rather than Lagrangians in relativistic theories of gravity Class. Quantum Grav. 25175024 (Preprint arXiv:0807.3079 [gr-qc])

[48] Bouchareb A and Clément G 2007 Black holes mass and angular momentum in topologically massive gravity Class. Quantum Grav. 245581 (Preprint arXiv:0706.0263 [gr-qc])

[49] Bondi H, Metzner A W K and Van der Berg M J C 1962 Gravitational waves in general relativity. VII. Waves from axi-symmetrical isolated systems Proc. R. Soc. A London 26921 
[50] Pitts J B, Gauge-invariant localization of many gravitational energies from all possible auxiliary structures (Preprint arXiv:0902.1288 [gr-qc])

[51] Chamseddine A H, 1990 Topological gravity and supergravity in various dimensions, Nucl. Phys. B 346213

[52] Regge T and Teitelboim T 1974 Role of surface integrals in the Hamiltonian formulation Ann. Phys. $\mathbf{8 8} 286$

\section{Erratum, submitted to CQG}

Petrov A N 2009 Three types of superpotentials for perturbations in the Einstein-Gauss-Bonnet gravity Class. Quantum Grav. 26135010 (16pp) (Paper the above.)

In this short communication we want to correct a mistake in our the above work. For the coefficient $m$ in (2.9) it was taken a wrong order of a covariant differentiation of $g$ in the first case (first line). The right writing is

$$
\begin{aligned}
\hat{m}_{\sigma}{ }^{\alpha \tau} & \equiv-\frac{1}{2 \kappa}\left\{\left.\left[\frac{\partial \hat{\mathcal{L}}_{c}}{\partial\left(\bar{D}_{\alpha} g^{a}\right)}-\bar{D}_{\beta}\left(\frac{\partial \hat{\mathcal{L}}_{c}}{\partial\left(\bar{D}_{\alpha} \bar{D}_{\beta} g^{a}\right)}\right)\right] g^{a}\right|_{\sigma} ^{\tau}\right. \\
& \left.-\frac{\partial \hat{\mathcal{L}}_{c}}{\partial\left(\bar{D}_{\tau} \bar{D}_{\alpha} g^{a}\right)} \bar{D}_{\sigma} g^{a}+\frac{\partial \hat{\mathcal{L}}_{c}}{\partial\left(\bar{D}_{\beta} \bar{D}_{\alpha} g^{a}\right)} \bar{D}_{\beta}\left(\left.g^{a}\right|_{\sigma} ^{\tau}\right)\right\} .
\end{aligned}
$$

In the result, some formulae of the canonical approach have to be changed. Thus instead of the formulae (3.2) and (3.3), which define the general canonical superpotential in EGB gravity, one must to write, respectively,

$$
\begin{aligned}
& \hat{\imath}_{C}^{\alpha \beta}=E^{\hat{\imath}_{C}^{\alpha \beta}}+{ }_{G B} \hat{\imath}_{C}^{\alpha \beta} \\
& =\frac{1}{\kappa}\left(\hat{g}^{\rho[\alpha} \bar{D}_{\rho} \xi^{\beta]}+\hat{g}^{\rho[\alpha} \Delta_{\rho \sigma}^{\beta]} \xi^{\sigma}\right) \\
& -\frac{2 \alpha \sqrt{-g}}{\kappa}\left\{\Delta_{\lambda \sigma}^{\rho} R_{\rho}^{\lambda \alpha \beta}+4 \Delta_{\lambda \sigma}^{\rho} g^{\lambda[\alpha} R_{\rho}^{\beta]}+\Delta_{\rho \sigma}^{[\alpha} g^{\beta] \rho} R\right\} \xi^{\sigma} \\
& -\frac{2 \alpha \sqrt{-g}}{\kappa}\left\{R_{\sigma}{ }^{\lambda \alpha \beta}+4 g^{\lambda[\alpha} R_{\sigma}^{\beta]}+\delta_{\sigma}^{[\alpha} g^{\beta] \lambda} R\right\} \bar{D}_{\lambda} \xi^{\sigma} \text {, } \\
& \overline{\hat{\imath}_{C}^{\alpha \beta}}=E^{\overline{\hat{\imath}_{C}^{\alpha \beta}}}+G_{B} \overline{\hat{\imath}_{C}^{\alpha \beta}} \\
& =\frac{1}{\kappa} \overline{D^{\left[\alpha \hat{\xi}^{\beta]}\right.}}-\frac{2 \alpha}{\kappa}\left\{\overline{\hat{R}}_{\sigma}^{\lambda \alpha \beta}+4 \overline{g^{\lambda[\alpha} \hat{R}_{\sigma}^{\beta]}}+\delta_{\sigma}^{[\alpha} \overline{g^{\beta] \lambda} \hat{R}}\right\} \bar{D}_{\lambda} \xi^{\sigma} .
\end{aligned}
$$

One can see that the mistake influences only on the GB part. Next, instead of the formulae (4.3), (4.4) and (4.5) one has the following formulae, respectively,

$$
\begin{aligned}
\hat{\imath}_{C}^{01} & +\kappa^{-1} \lambda^{[0} \hat{d}_{D K O}^{1]}=\frac{\sqrt{-\bar{g}}}{2 \kappa r}\left[\frac{r \bar{f}^{\prime}}{2}\left(\frac{f}{\bar{f}}+\frac{\bar{f}}{f}\right)-(f-\bar{f})(D-2)\right] \\
& +\frac{\alpha \sqrt{-\bar{g}}}{\kappa r^{2}}(D-2)(f-\bar{f})\left(f^{\prime}-r f^{\prime \prime}\right) \\
& -\frac{\alpha \sqrt{-\bar{g}}}{\kappa r^{3}}(D-2)(D-3)(f-1)\left[\frac{r \bar{f}^{\prime}}{2}\left(\frac{f}{\bar{f}}+\frac{\bar{f}}{f}\right)-(f-\bar{f})(D-2)\right],
\end{aligned}
$$




$$
\begin{gathered}
\hat{\imath}_{C}^{01}+\kappa^{-1} \lambda^{[0} \hat{d}_{K L}^{1]}=\frac{\sqrt{-\bar{g}}}{2 \kappa r}\left[\frac{r \bar{f}^{\prime}}{2}\left(\frac{f}{\bar{f}}+\frac{\bar{f}}{f}\right)-(f-\bar{f})(D-2)\right] \\
+\frac{\alpha \sqrt{-\bar{g}}}{\kappa r^{2}}(D-2)(D-3)(f-\bar{f}) f^{\prime} \\
-\frac{\alpha \sqrt{-\bar{g}}}{\kappa r^{3}}(D-2)(D-3)(f-1)\left[\frac{r \bar{f}^{\prime}}{2}\left(\frac{f}{\bar{f}}+\frac{\bar{f}}{f}\right)-(f-\bar{f})(D-4)\right], \\
\overline{\hat{\imath}_{C}^{01}}=\frac{\sqrt{-\bar{g}}}{2 \kappa} \bar{f}^{\prime}-\frac{\alpha \sqrt{-\bar{g}}}{\kappa r^{2}}(D-2)(D-3) \bar{f}^{\prime}(\bar{f}-1) .
\end{gathered}
$$

The above three expressions define the canonical superpotential for the static spherically symmetric solutions of the Schwarzschild-like form.

Turn to the formula (5.6), which is used for calculating the mass of the Schwarzschild-anti-de Sitter black hole. It has a place for all the three approaches examined in the paper and is the main paint in carrying out the applications. It turns out that this asymptotic formula is conserved for the here corrected canonical superpotential in the EGB gravity. Inversely, it is surprising that (5.6) has a place for the previous wrong canonical superpotential! Also, the mistake has no an influence on writing the Belinfante superpotential because it does not depend on $m$-coefficient; and the mistake has no an influence on writing the symmetrical superpotential because it depends on the coefficient $N$ in (2.21), where the order of particular derivatives of $g$ is not important. At last, we note that, in spite of the mistake, all the discussions and conclusions of the paper are right.

To conclude the comment we remark the following. How we understand now, after analyzing the mistake, there exist different possibilities to choose an order of covariant derivatives of $g$ in definitions of coefficients, like $m$ in (2.9) and $n$ in (2.10). In the other words, the choice in $n$ defines the choice in $m$. Only, in all the cases $n$ and $m$ have to satisfy the standard system of the Nœther identities. In future, we plan to study this possibility constructing a new set of conserved quantities (including new set of superpotentials) by a non-contradictive way. 